\title{
TABLE OF CASES AND AWARDS
}

\section{COURT OF JUSTICE OF THE EUROPEAN UNION (CJEU)}

C-29/76, LTU Lufttansportunternehmen GmbH \& Co. KG v Eurocontrol [1976] E.C.R.

1541 (C-29/76, LTU)

1.34

C-814/79, Netherlands v Rüffer [1980] E.C.R. 3807

1.34

C-145/86, Hoffmann v Krieg [1988] E.C.R. 645 (C-145/86, Hoffmann) $7.16,7.18$

C-81/87, Daily Mail v UK [1988] E.C.R 5483 4.13

C-190/89, Marc Rich E Co. AG v Società Italiana Impianti PA [1991] E.C.R. I-3855

(C-190/89, Marc Rich) $1.18,2.11,2.13,2.16,2.19,2.21,2.26,2.31$

C-351/89, Overseas Union v New Hampshire Insurance Co [1991] E.C.R. I-3342

(C-351/89, Overseas Union) $6.13,6.19$

C-26/91, Jakob Handte \& Co GmbH v Traitements Mécano-chimiques des Surfaces SA

[1992], E.C.R. I-3967 3.71

C-89/91, Shearson Lebman Hutton v TVB Treuhandgesellschaft für Vermögensverwaltung und

Beteiligungen GmbH [1993] E.C.R. I-139 1.16

C-172/91, Sonntag v Waidmann [1993] E.C.R. I-1963 1.34

C-129/92, Owens Bank Ltd v Fulvio Bracco and Bracco Industria Chimica SpA [1994],

E.C.R. I- 117 (C-129/92, Owens Bank) $3.71,6.19$

C-288/92, Custom Made Commercial Ltd v Stawa Metallbau GmbH [1994] E.C.R.

I-2913 .3 .71

C-414/92, Solo Kleinmotoren GmbH v Emilio Boch [1994] E.C.R. I-2247 (C-414/92,

Solo Kleinmotoren) $3.16,7.10,7.11,7.12$

C-406/92, The owners of the cargo lately laden on board the ship 'Tatry' and the owners of the

ship 'Maciej Rataj' [1994] E.C.R. I-5439 (C-406/92, Tatry) $6.14,6.15$

C-269/95, Francesco Benincasa v Dentalkit Srl [1997] E.C.R. I-3767 .3 .71

C-391/95, Van Uden Maritime BV, trading as Van Uden Africa Line v

Kommanditgesellschaft in Firma Deco-Line [1998] E.C.R. I-7091 (C-391/95, Van

Uden) $1.18,2.11,2.13,2.16,2.19,2.26,2.31$

C- 351/96, Douot Assurances SA v CMI [1998] E.C.R. I-3075 6.14

C-51/97, Réunion européenne SA and Others v Spliethoff's Bevrachtingskantoor BV and the

Master of the vessel 'Alblasgracht V002' [1998] E.C.R. I-6511 ..................................... 3.71

C-90/97, Robin Swaddling v Adjudication Officer [1999] E.C.R. I-01075 ..............................37

C-126/97, ECO Swiss v Benetton [1999] E.C.R. I-3079 (C-126/97, ECO Swiss) ............. 3.23

C-212/97, Centros [1999] E.C.R. I-1459 ...........................................................................13

C-440/97, GIE Groupe Concorde and Others v The Master of the vessel 'Suhadiwarno

Panja' and Others [1999] E.C.R. I-6307

C-7/98, Krombach v Bamberski, [2000] E.C.R. I-1935 (C-7/98, Krombach).... 3.20, 3.21, 3.22,

$3.23,7.27,7.28$

C-38/98, Régie Nationale des Usines Renault SA v Maxicar SpA, Orazio Formento, [2000]

E.C.R. I-3009 (C-38/98, Renault v Maxicar) $3.22,3.23,7.28$

C-381/98, Ingmar GB Ltd. v Eaton Leonard Technologies Inc., [2000] E.C.R. I-09305 ..... 5.07

C-80/00, Italian Leather v WECO [2002] E.C.R. I-5011 7.17 
C-208/00, Überseering [2002] E.C.R. I-9919 4.13

C-167/01, Inspire Art [2003] E.C.R. I-10155 4.13

C-39/02, Marsk Olie E Gas A/S v Firma M. de Haan en W. De Boer [2004] E.C.R.

I-9657 .6 .14

C-116/02, Gasser MnbH v Misat Srl [2003] E.C.R. I-14721 (C-116/02, Gasser) ... 3.72, 6.16,

C-159/02, Gregory Paul Turner v Felix Fareed Ismail Grovit and Others [2004] E.C.R.

I-3565 (C-159/02, Turner v Grovit) $2.18,3.72$

C-281/02, Andrew Owusu v N.B. Jackson, trading as 'Villa Holidays Bal-Inn Villas' and

Others [2005] E.C.R. I-1383 (C-281/02, Owusu) $1.35,3.71$

C-216/06, Cartesio [2008] E.C.R. I-9641

C-185/07, Allianz SpA v West Tankers [2009] E.C.R. I-00663 (C-185/07, West Tankers) ..1.18

C-167/08, Draka NK Cables Ltd, AB Sandvik International, VO Sembodja BV, Parc

Healthcare International Ltd v Omnipol Ltd [2009] E.C.R. I-3477 1.17

C-189/08, Zuid-Chemie BV v Philippo's Mineralenfabriek NV/SA [2009] E.C.R.

I-6917

C-292/08, German Graphics Graphische Maschinen GmbH v Alice van der Schee [2009]

E.C.R. I-8421

C-533/08, TNT Express Nederland BV $\mathrm{v} A X A$ Versicherung $A G$, [2010] E.C.R.

I-04107

C-523/10, Wintersteiger $A G \mathrm{v}$ Products $4 U$ Sondermaschinenbau GmbH [2012]

ECLI:EU:C:2012:220

C-133/11, Folien Fischer $A G$ v Ritrama SpA [2012] ECLI:EU:2012:664

C- 456/11, Gothaer Allgemeine versicherung AG v Samskip GmbH [2012]

ECLI:EU:C:2012:719

C-536/13, Gazprom OAO v Lietuvos Respublika, [2015] EU:C:2015:316 (C-536/13,

Gazprom) $2.22,2.23,2.26,2.27,2.28,2.32,3.36,3.40$

\section{NATIONAL COURTS}

\section{England}

\section{Supreme Court/House of Lords}

Black Clawson International Ltd. v Papierwerke Waldhof-Aschaffenberg AG [1981] 2 Lloyd's

Rep. 446 4.158

Carl Zeiss Stiftung Appellants v Rayner E Keeler Ltd. [1967] 1 A.C. 853 7.62

Channel Tunnel Group Ltd. v Balfour Beauty Construction Ltd. [1993] A.C. 334 4.158

Showlag v Mansour [1995] 1 A.C. 431 (P.C.)

\section{Court of Appeal}

Ahmad Al-Naimi v Islamic Press Agency Inc. [2000] 1 Lloyd's Rep. 522 (Ahmad Al-Naimi [2000] 1 Lloyd's Rep. 522) 6.100, 6.101, 6.109

$C$ v $D$ [2008] 1 Lloyd's Rep. 239 4.161, 4.162

ET Plus SA v Jean-Paul Welter [2005] EWHC 2115 
Fulham Football Club v Richards [2011] EWCA Civ 855 (Fulham Football Club [2011]

EWCA Civ 855) $5.73,5.74,5.76$

Good Challenger Navegate SA v Metalexportimport SA [2003] EWCA Civ 1668 7.62

Naviera Amazonica Peruana SA v Cia Internacional de Seguros del Peru [1998] 1 Lloyd's Rep.

116 4.156

National Navigation v Endesa Generacion SA [2009] EWCA Civ 1397 (National Navigation

[2009] EWCA Civ 1397) $2.20,2.39,2.41,7.65,7.66$

Westacre Investments Inc. v Jugoimport-SDPR Holding Co. Ltd. [1999] 3 All E.R. 864 ...... 5.69

\section{High Court}

$A$ v $B$ [2007] 1 Lloyd's Rep. 237 (Comm.) 6.109

Cia Maritima Zorroza SA v Sesostris SAE [1984] 1 Lloyd's Rep. 652 4.157

Elektrim SA v Vivendi Universal SA [2007] 2 Lloyd's Rep. 8 (Comm.) 6.106

ET Plus SA v Jean-Paul Welter [2005] EWHC 2115 5.76

Leibinger and another $v$ Stryker Trauma GmbH [2006] EWHC 690 (Comm) (Leibinger v Stryker [2006] EWHC 690) . 4.157, 4.160

Shauhoua v Sharma [2009] 2 Lloyd's Rep. 376 (Comm.) 6.105

Sheffield United FC Ltd v West Ham United FC plc [2009] 1 Lloyd's Rep. 167

(Comm.) 6.107

Tamil Nadu Electricity Board v ST-CMS Electric Co. Ovt Ltd. [2007] EWHC 1713 (Comm.) 4.156

Union of India v McDonnell Douglas Corporation [1993] 2 Lloyd's Rep. 48 4.157

XL Insurance Ltd. v Owens Corning, [2000] 2 Lloyds Rep. 500 (Comm.) (XL Insurance [2000] 2 Lloyds Rep. 500) $4.158,4.159,4.160$

\section{France}

\section{Supreme Court (Cour de Cassation)}

Comité populaire de la municipalité de Khoms El Mergeb v Dalico Contractors, Dec. 1993, Revue de l'Arbitrage 116 (1994) 4.168

Hilmarton v Omnium de Traitement et de Valorisation, March 1994, Revue de l'Arbitrage 327 (1994) in Y.B Com. Arb., Vol. XX p. 663 $7.69,7.71,7.74$

M. Zanzi v J.de Coninck et autres, Jan. 1999, Revue de l'Arbitrage 260 (1999) (Zanzi,

Rev. Arb. 1999.260) 4.171

Omnium de Traitement et de Valorisation v Hilmarton, June 1997, 1997 Revue de

l'Arbitrage 376 (1997) in Y.B. Com. Arb., Vol. XXII, p. 665

Putrabali Adyamulia v Rena Holding, (two decisions) June 2007, note E. Gaillard in Y.B.

Comm. Arb., Vol. XXXII p. 299 $7.71,7.74$

Société $G$ et A Distribution SARL v société Prodim, société par actions simplifiée, May 2008,

Revue de l'Arbitrage 461 (2008), note L. Weiller 7.68

Société Uni-Kod v Société Ouralkali, Mar. 2004, Revue de l'Arbitrage 959 (2005) 4.172

SPP v Egypt (Cour de Cassation), Jan. 1987, Revue de l'Arbitrage 469 (1987), note Ph.

Leboulanger 4.176 


\section{Court of Appeal (Cour d'appel)}

Grenoble CFTE v Dechavanne, (Cour d'appel de Grenoble), Sep 1993, Revue de

l'Arbitrage 337 (1994), note M. A. Moreau

SA France Embryon v Renato Argonauta (Cour d'appel Lyon), Jul. 1991, in Y.B. Com. Arb.,

Vol. XIX, p. 859

Société Ganz et autres v. Société Nationale des Chemins de Fer Tunisiens (SNCFT) (Cour

d'appel de Paris), Mar. 1991, Revue de l'Arbitrage 478 (1991), note L. Idot ... 5.77, 5.78

Société KFTCIC v Société Icori Estero et autre, Cour d'appel de Paris (1Ch. C), Jun. 1996,

Revue de l'Arbitrage 251 (1997)

Société Murgue Seigle v société Coflexip (Cour d'appel de Paris), Mar. 1989, Revue de

l'Arbitrage 345 (1991)

SPP v Egypt, (Cour d'appel de Paris), Jul. 1984, Journal Droit International 129

(1985)

Sté Labinal v Sté Mors (Cour d'appel de Paris), May 1993, Revue de l'Arbitrage 645

(1993), note C. Jarrosson

\section{Germany}

\section{Supreme Court (Bundesgerichtshof)}

Saudi Arabian Party v German Party, Jun. 2002, case no: III ZB 44/01 in UNCITRAL's

CLOUT database (S. Kröll): case no. 560, UN Doc: A/CN.9/SER.C/

ABSTRACTS/49. (Saudi Arabian Party v German Party, Bundesgerichtshof, case no: III

ZB 44/01(2002)) $6.35,6.79$

\section{Courts of Appeal/Higher Regional Courts (Oberlandesgericht)}

Bayerische Oberste Landesgericht (Bavarian) decision case no.: 4 Z SchH 03/99, Sep. 1999, available at www.dis-arb.de 5.56

Buyer (Poland) v Seller (Poland) Hanseatisches Oberlandesgericht (Hamburg), case no.: $11 \mathrm{SCH}$ 06/01, Jan. 2003, in in Y.B. Com. Arb., Vol. XXX, p. 516 4.150

German manufacturer v Dutch distributor, Landgericht of Heidelberg, Oct. 1972, affirmed by the Oberlandesgericht Karlsruhe, Mar. 1973, in Y.B. Com. Arb., Vol. II, p. 239 ....... 4.106

Italy

\section{Supreme Court (Corte di Cassazione)}

Il Nuovo Castoro v Italian Ministry of Foreign Affairs, Oct. 2000, in Y.B. Com. Arb., Vol.

XXVI p. 1141

\section{Courts of First Instance (Tribunale)}

Adda Officine Elettromeccaniche e Meccaniche, Ercole Marelli \& C. SpA v Alsthom International

SA and others, (Tribunale Lodi), Feb. 1991, in Y.B. Com. Arb., Vol. XXI, p. 580 ... 4.106 


\section{Singapore}

PT Asuransi Jasa Indonesia (Persero) v Dexia Bank SA, Singapore Court of Appeal, Dec. 2006, case no: SGCA 41 in UNCITRAL's CLOUT database (L. Boo), case no. 742, A/CN.9/SER.C/ABSTRACTS/70

\section{Spain}

\section{Courts of Appeal (Audiencia Provincial)}

Licensing Projects SL (Spain) v Pirelli \& C. SpA (Italy), Barcelona Audiencia Provincial, Case no. 86/2009 (Section 15), Apr. 2009, in Y.B. Com. Arb., Vol. XXXV p. 452

\section{Sweden}

\section{Swedish Supreme Court (Högsta Domstolen)}

Bulgarian Foreign Trade Bank Ltd. (Bulbank) v A. I. Trade Finance (AIT) (NJA 2000 p. 538)

Esselte AB v Allmänna Pensionsfonden (NJA 1998 p. 189) (Esselte AB (NJA 1998

$$
\text { p. 189)) }
$$

\section{Svea Court of Appeal (Svea Hovrätt)}

Czech Republic v CME Czech Republic BV, case no. T-8735-01 (RH 2003:55)

\section{Switzerland}

\section{Supreme Court (Tribunal Fédéral)}

Club Atletico de Madrid SAD v Sport Lisboa e Benfica Futebol SAD, Apr. 2010, available at www.bger.ch (accessed Jan. 2016)

\section{USA}

\section{Supreme Court}

Mitsubishi Motors Corp. v Soler Chrysler-Plymouth Inc. 473 U.S. 614 (1985) (Mitsubishi Motors 473 U.S. 614 (1985))

\section{Federal courts}

Parsons E Whittemore Overseas Co. Inc. v Société Générale de l'Industrie du Papier (Rakta) 508 F.2d 9691974 (2d Cir.) (Overseas v Rakta 508 F.2d 9691974 


\section{Zimbabwe}

Zimbabwe /Harare High Court, Durco (Pvt) Ltd v Dajen (Pvt) Ltd, Jul. and Aug. 1997, case no. HH-133-97 in UNCITRAL CLOUT database, case no. 233, A/CN.9/SER.C/ABSTRACTS/20 7.51

\section{ARBITRAL TRIBUNALS}

Ad hoc arbitration No. 27, Alpha Inc v Beta Corp., preliminary award on jurisdiction rendered in 1992 in Geneva in ASA Bull. 481 (1994)

Arbitration Court of the Bulgarian Chamber of Commerce and Industry, Case No. 80/1970, Bulgarian State enterprise v Italian enterprise in Y.B. Com. Arb., Vol. IV, p. 190

Hamburger Freundschaftliche Arbitrage, German seller v Dutch buyer in Y.B. Com. Arb., Vol. III, p. 212 $4.17,4.122$

ICC Award No. 1512/1970, Indian Cement Company v Pakistani Bank in Y.B. Com. Arb., Vol. V, p. 174 4.76

ICC Award No. 1803/1972, Société des Grands Travaux de Marseille v East Pakistan Industrial Development Corporation in Y.B. Com. Arb., Vol. V, p. 180

ICC Award No. 4145/1983, Establishment of Middle East country v South Asian construction company in Y.B. Com. Arb., Vol. XII, p. 97

ICC Award No. 4604/1984, Italian company v US company in 112 Journal Droit International 973 (1985)

ICC Award No. 4695/1984, Parties from Brazil, Panama and USA v Party from Brazil in Y.B. Com. Arb., Vol. XI, p. 151

ICC Award No. 5065/1986, Lebanese Party v two Pakistani companies in 114 Journal Droit International 1039 (1987)

ICC Case No. 4131/ 1984, Dow Chemical France, The DOW Chemical Co. and others v Isover Saint Gobain in Y.B. Com. Arb., Vol. IX, p. 133 (Dow Chemical, Y.B. Com. Arb., Vol. IX, p. 133)

ICC Case No. 6531/1991, in Y.B. Com. Arb., Vol. XVII, p. 221 $4.25,4.27$

ICC Partial Award No. 6697/1990, Casa v Cambior, Revue d l'Arbitrage 135 (1992) ..... 5.84

Netherlands Oils, Fats and Oilseeds Trade Association, award (1977), Dutch private company v Tunisian private company in Y.B. Com. Arb., Vol. III, p. 225 